\title{
Dynamic assessment of parathyroid function in acute malaria
}

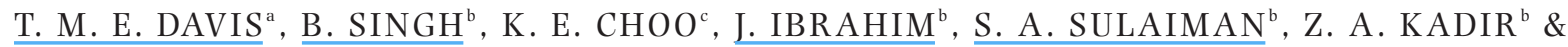 \\ R. ISMAIL ${ }^{\mathrm{d}}$ \\ ${ }^{\mathrm{a}}$ University of Western Australia, Department of Medicine, Fremantle Hospital, Fremantle, Western Australia, Australia. ${ }^{\mathrm{b}}$ School of Medical Sciences, \\ Universiti Sains Malaysia, Kubang Kerian, Kota Bharu; 'Department of Paediatrics, General Hospital, Kota Bharu, and ${ }^{\mathrm{d}}$ Kuala Krai Hospital, Kuala \\ Krai; Kelantan, Malaysia
}

\begin{abstract}
Davis TME, Singh B, Choo KE, Ibrahim J, Sulaiman SA, Kadir ZA, Ismail R (University of Western Australia, Fremantle Hospital, Fremantle, Western Australia, Australia. Universiti Sains Malaysia, Kubang Kerian, Kota Bharu; General Hospital, Kota Bharu, Kuala Krai Hospital, Kuala Krai; Kelantan, Malaysia) Dynamic assessment of parathyroid function in acute malaria. J Intern Med 1998; 243: 349-54.
\end{abstract}

Objectives. To investigate the dynamic parathyroid response to rapidly induced, sustained hypocalcaemia in patients with acute malaria and in healthy volunteers.

Design. Serum intact parathormone (PTH) concentrations were measured on samples taken before and during a variable-rate tri-sodium citrate infusion designed to 'clamp' the whole blood ionised calcium concentration $0.20 \mathrm{mmol} \mathrm{L}^{-1}$ below baseline for 120 min.

Subjects. Six Malaysian patients aged 17-42 years with acute malaria, four of whom were restudied in convalescence, and 12 healthy controls aged 19-36 years.

Main outcome measures. Whole-blood ionised calcium and serum intact PTH concentrations.

Results. The mean (SD baseline ionised calcium was lower in the malaria patients than in controls (1.09 \pm 0.06 vs. $1.18 \pm 0.03 \mathrm{mmol} \mathrm{L}^{-1}$, respectively; $P=$
0.01) but PTH concentrations were similar (3.0 \pm 1.8 vs. $\left.3.3 \pm 1.3 \mathrm{pmol} \mathrm{L}^{-1} ; P=0.33\right)$. Target wholeblood ionised calcium concentrations were achieved more rapidly in the controls than the patients (within 15 vs. $30 \mathrm{~min}$ ) despite significantly more citrate being required in the patients (area under the citrate infusion-time curve 0.95 (0.25 vs. $0.57 \pm 0.09$ mmol kg-1 $P<0.01$ ). The ratio of the change in serum PTH to that in ionised calcium $\left(\Delta \mathrm{PTH} / \Delta \mathrm{Ca}^{2+}\right)$, calculated to adjust for differences in initial rate of fall of ionised calcium, was similar during the first 5 min of the clamp $\left(132 \pm 75 \times 10^{-6}\right.$ vs. $131 \pm 43 \times$ $10^{-6}$ in patients and controls, respectively, $P>0.05$ ), as were steady-state serum PTH levels during the second hour $\left(7.0 \pm 2.2 \mathrm{pmol} \mathrm{L}^{-1}\right.$ in each case). Convalescent patients had normal basal ionised calcium levels but the lowest serum intact PTH levels before and during the clamp, consistent with an increase in skeletal PTH sensitivity after treatment.

Conclusions. There is a decreased ionised calcium 'set point' for basal PTH secretion but a normal PTH response to acute hypocalcaemia in malaria. Skeletal resistance may attenuate the effects of the PTH response but patients with malaria appear relatively resistant to the calcium chelating effects of citrated blood products.

Keywords: citrate clamp, hypocalcaemia, malaria, parathormone.

\section{Introduction}

Parathyroid hormone (PTH) secretion is regulated by the extracellular ionised calcium concentration in a sigmoid relationship [1]. The 'set point' of the sigmoid curve, where half maximal inhibition of PTH secretion occurs, is within or close to the wholeblood ionised calcium concentration in healthy vol- unteers [2]. The combination of mild hypocalcaemia and a normal serum intact PTH concentration occurs commonly in patients with uncomplicated malaria and in severe cases without renal impairment [3-5]. This suggests that there is a lower set point for PTH secretion, with the sigmoid curve shifted to the left.

When renal failure complicates acute malaria, 